\title{
PointCloudXplore 2: Visual Exploration of 3D Gene Expression
}

\author{
O. Rübel ${ }^{1,2,3}$, G.H. Weber ${ }^{3}$, M.-Y. Huang ${ }^{2}$, E.W. Bethel ${ }^{3}$, S.V.E. Keränen ${ }^{4}$, \\ C.C. Fowlkes ${ }^{5}$, C.L. Luengo Hendriks ${ }^{7}$, Angela H. DePace ${ }^{8}$, L. Simirenko ${ }^{4}$, \\ M.B. Eisen ${ }^{4}$, M.D. Biggin ${ }^{4}$, H. Hagen ${ }^{1}$, J. Malik ${ }^{6}$, \\ D.W. Knowles ${ }^{7}$ and B. Hamann ${ }^{1,2,3}$
}

${ }^{1}$ International Research Training Group Visualization of Large and Unstructured Data Sets, University of Kaiserslautern, Germany

${ }^{2}$ Institute for Data Analysis and Visualization, University of California, Davis, CA

${ }^{3}$ Computational Research Division, Lawrence Berkeley National Laboratory

(LBNL), Berkeley, CA

${ }^{4}$ Genomics Division, LBNL

${ }^{5}$ Computer Science Department, University of California, Irvine, CA

${ }^{6}$ Computer Science Division,University of California, Berkeley, CA

${ }^{7}$ Life Sciences Division, LBNL

${ }^{8}$ Department of Molecular and Cellular Biology and the Center for Integrative Genomics, University of California, Berkeley, CA

\begin{abstract}
To better understand how developmental regulatory networks are defined in the genome sequence, the Berkeley Drosophila Transcription Network Project (BD$N T P$ ) has developed a suite of methods to describe 3D gene expression data, i.e., the output of the network at cellular resolution for multiple time points. To allow researchers to explore these novel data sets we have developed PointCloudXplore (PCX). In PCX we have linked physical and information visualization views via the concept of brushing (cell selection). For each view dedicated operations for performing selection of cells are available. In PCX, all cell selections are stored in a central management system. Cells selected in one view can in this way be highlighted in any view allowing further cell subset properties to be determined. Complex cell queries can be defined by combining different cell selections using logical operations such as AND, OR, and NOT. Here we are going to provide an overview of PointCloudXplore 2 (PCX2), the latest publicly available version of PCX. PCX2 has shown to be an effective tool for visual exploration of 3D gene expression data. We discuss (i) all views available in PCX2, (ii) different strategies to perform cell selection, (iii) the basic architecture of PCX2., and (iv) illustrate the usefulness of PCX2 using selected examples.
\end{abstract}




\section{Introduction}

Understanding how embryo development is controlled is a fundamental question in biology. Specific combinations of developmental regulatory factors -forming parts of complex genetic regulatory networks- are responsible for determining the cell fates [Law92]. Biologists have typically analyzed gene expression and morphology by visual inspection of photomicrographic images. In order to understand animal development, methods to computationally describe gene expression at cellular resolution are required. To address this challenge, the BDTNP has developed image analysis methods to extract information about gene expression from 3D imaging data, using early Drosophila melanogaster embryos as a model [BDT]. Blastoderm stage Drosophila embryos are stained, mounted, and imaged using laser microscopy. The image stacks are then converted into matrices specifying the position of blastoderm nuclei and the expression levels of select genes in and around each nucleus $\left[\mathrm{LKF}^{+}\right.$06, $\mathrm{KFL}^{+}$06]. These novel, so called PointCloud datasets, promise to be an invaluable resource for studying embryo development. Due to the limited number of different fluorophores we can spectrally distinguish as well as the difficulty in adding multiple labels to embryos it is, however, experimentally not practical to obtain the expression of more than a few genes in a single embryo. To make possible to compare the relationships between regulators and their many target genes in a common coordinate framework, a set of PointClouds are registered into one or more Virtual PointClouds $\left[\mathrm{FLK}^{+}\right.$05, $\mathrm{FLK}^{+}$07].

To help biologists to compare and analyze 3D gene expression data, we developed PointCloudXplore (PCX), a tool specifically designed for exploration of PointCloud data. In PCX, we have linked physical and information visualization views via the concept of brushing (cell selection). Here we are going to introduce PointCloudXplore 2 (PCX2), the next publicly available version of PCX. We are going to discuss all views available in PCX2 and describe how linking of the views via cell selection is used for effective data exploration. Afterwards, we will explain different dedicated strategies for performing cell selection and describe the basic architecture of PCX2. The following parts of this manuscript are structured as follows. In Section 2 we will provide an overview of related work. The different visualization techniques available in PCX2 are then described in Section 3. In Section 4.1 and 4.2, linking of the views via cell selection and all operations for performing and combining cell selections will be explained. A brief overview of the implementation of PCX2 will be provided in Section 5. In Section 6 we present our conclusions and describe future plans.

\section{Previous Work}

PCX2 makes use of the established concept of linking multiple views to visualize highdimensional 3D gene expression data [BWK00]. In the WEAVE system, e.g., a combination of linked physical views and information visualization views was used for exploration of cardiac simulation and measurement data $\left[\mathrm{GRW}^{+} 00\right]$. Henze [Hen98] proposed a system of multiple views (called Portraits in his paper) for exploring time-varying computational fluid dynamics (CFD) data sets. Both Henze's system for CFD data and the 
WEAVE system use linked views to define features in a data set by refining queries based on brushes. In PCX2, brushes are called cell selectors since this term is found to be more intuitive by target users. Doleisch et al. formalized the concept of defining features via queries using information visualization views and utilizing logical operations to combine several brushes [DGH03] (see also Hauser et al. [HLD02]). A variety of extensions to 3D scatter plots were proposed by Piringer et al. [PKH04] and Kosara et al. [KSH04] improving depth perception and perception of the sample distribution in all dimensions.

Parallel coordinates are a standard visualization for high-dimensional data while many extensions to standard parallel coordinates have been developed to make them more useful. Wegman and Luor [WL97] proposed usage of transparency and "over-plotting" translucent data points/lines to reveal inherent data characteristics by highlighting dense areas. Fua et al. [FWR99] proposed hierarchical parallel coordinates, including several techniques for visualization of selected subsets of the data which are in part also available in PCX2.

\section{Visualizing 3D Gene Expression}

PCX2 provides different visualizations for 3D gene expression data. In physical views a 3D model, or 2D projections of the 3D model, are used to visualize spatial gene expression patterns (Section 3.1). To support analysis of relationships between the expression of genes directly in expression space, PCX2 features scatter-plots (Section 3.2) as well as parallel coordinates (Section 3.3) as abstract information visualization views. To allow comparison of the expression of genes in a single cell, the Cell Magnifier shows the expression of all genes in a user defined cell as bar-graph (Section 3.4).

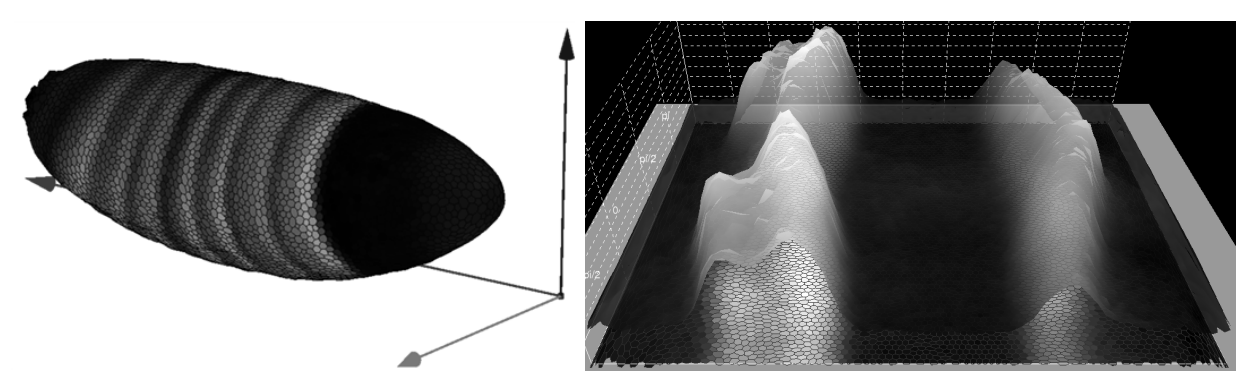

Figure 1: 3D Cell View with expression levels of the genes eve and $\mathrm{ftz}$ shown via color (left). Unrolled View and expression surface showing the expression pattern of the gene giant $(g t)$ during early embryo development (right).

\subsection{Physical Views}

Physical views are used in PCX2 to visualize and analyze spatial patterns of gene expression. PCX2 features $3 \mathrm{D}$ as well as $2 \mathrm{D}$ visualizations of the embryo. In the $3 \mathrm{D}$ views 
spherical icons (not shown) or polygonal faces are used to represent individual cells (see Figure 1 left). To also take the movement of cells over time into account the user can choose which cell positions from which time-step should be used in the visualization. By using different projections of the 3D embryo model dedicated 2D visualizations of the embryo are created. Figure 1 (right) shows an embryo projected to a $2 \mathrm{D}$ plane using a cylindrical projection. PCX2 also allows one to use orthographic projection to create three additional 2D views of the embryo described in more detail in Weber et al. [ $\left.\mathrm{WRH}^{+} 07\right]$.

In addition to providing an overview of the entire embryo, projecting the embryo to a plane has the advantage of freeing one dimension up for displaying additional information. In PCX2, this "free" dimension can be used to display gene expression values as surface plots. The height of an expression surface is determined by the expression values measured for the gene it represents while the $x y$-position of surface points are defined by the position of the cells in the underlying 2D physical view. As shown in the right panel of Figure 1, such surface plots enable a more quantitative analysis of gene expression data than possible when just using color. Multiple surfaces can be used to compare quantitative relationships between genes. More details about physical views and their use for the analysis of 3D gene expression are provided in $\left[\mathrm{RWK}^{+} 06, \mathrm{WRH}^{+} 07\right]$.

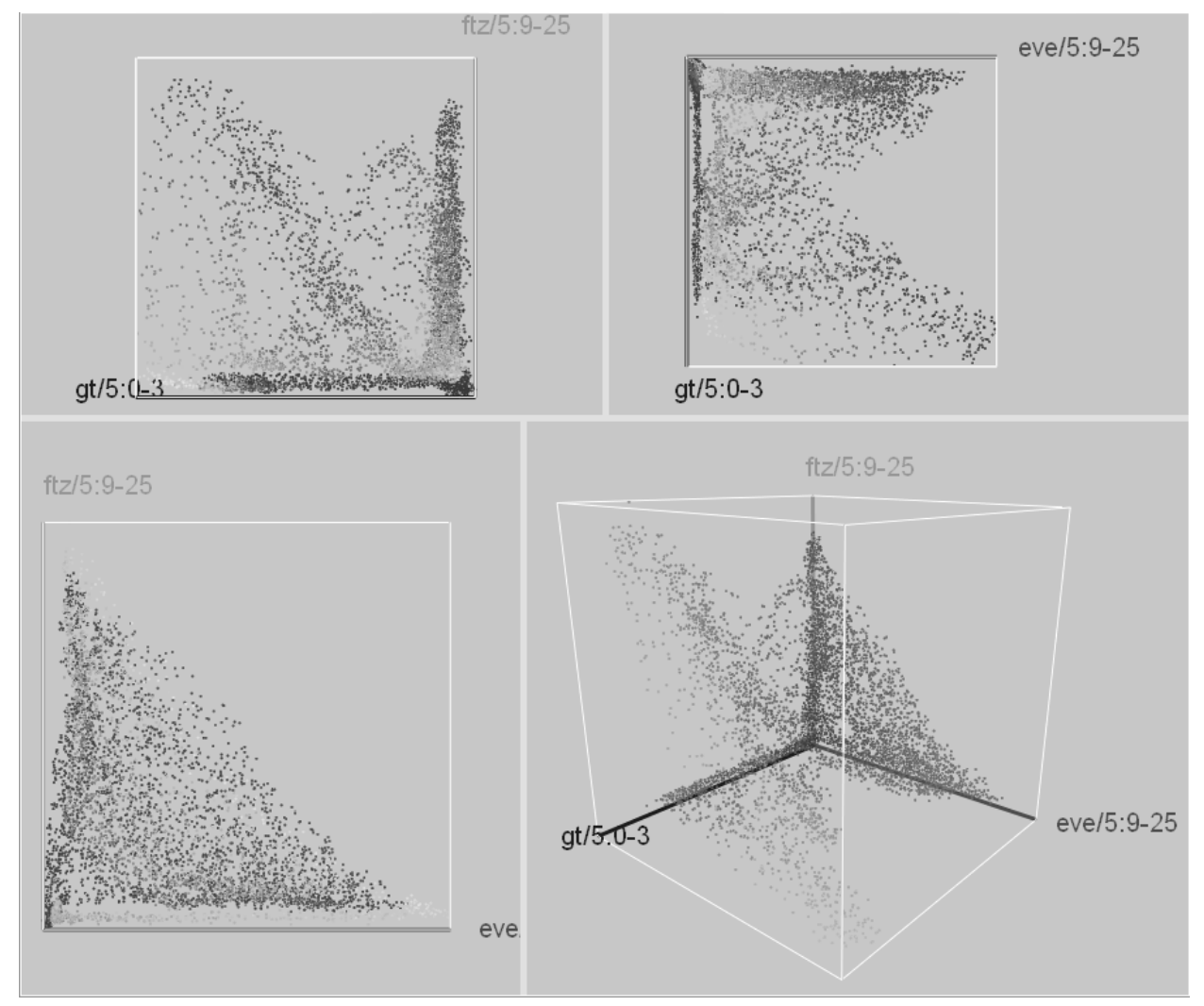

Figure 2: 4-panel layout of three 2D and the according 3D scatter-plot as displayed in PCX2 


\subsection{Scatter-plots}

In contrast to physical views of the embryo, abstract views, such as scatter-plots, discard spatial information and show the quantitative relationships between multiple genes expression in one or all cells. In a scatter-plot, three genes are selected and mapped to the three axes of the Cartesian coordinate system. Each of the axes represents the expression of one gene ranging from no expression at the origin to maximum relative expression. A single point is used to represent each cell of the embryo while the location of the point is specified according to the relative gene expression levels measured for the corresponding cell. To enhance depth perception and to better distinguish separate points we use color, halos, and alpha blending. As shown in Figure 2, in PCX2 we use a 4-panel layout in which a $3 \mathrm{D}$ scatter-plot (lower-right panel) is augmented with a set of 2D scatter plots showing the expression relationships between the three possible gene-pairs in the 3D plot. See Weber et al. [WRH ${ }^{+}$07] for more detailed information.

Scatter-plots are in general found to be an intuitive visualization and are used to reveal information about relationships between genes. In the scatter-plot of eve and $\mathrm{ftz}$ (Figure 2 lower-left panel), e.g., a clear anti-correlation between the two genes can be seen.

\subsection{Parallel Coordinates}

To make visualization of the expression of many genes in parallel possible, PCX2 features parallel coordinates. Parallel coordinates were proposed independently by Inselberg [Ins84] and Wegman [Weg90] and are a common information visualization technique for high-dimensional data sets. Each data dimension (gene) is represented in parallel coordinates by one parallel axis. Each data sample (cell) has a set of associated expression values defining a point on each parallel axis. By connecting the corresponding points of neighboring axes each cell can be represented by a data line, i.e., a "zigzag" line connecting adjacent parallel axes. The intersection point of the data line with each vertical axis corresponds to the value of the sample for the corresponding dimension (i.e., the relative expression level for the corresponding gene in that cell). In Figure 3, an example parallel coordinate view is shown visualizing the expression of six selected genes in all cells of the embryo.

3D-parallel coordinates are a direct extension of 2D-parallel coordinates in which the parallel axes are extruded into the $z$ dimension. As illustrated in Figure 4, lines are sorted along the $z$ axis with respect to a selected data dimension so that each parallel axis is de facto a 2D-scatter-plot. As in 2D-parallel coordinates, corresponding points of neighboring axes are connected via line segments. In PCX2 the same information is displayed along the $z$ dimension for all axes, in this way a 3D-plot of non-intersecting parallel data lines is created each representing one cell. Data lines can be sorted in parallel coordinates according to the position of cells along the anterior-posterior axis (AP)(head to rear) or dorsal-ventral circumference (DV)(circumference around the embryo) which can be derived from the Unrolled View. Beside this, lines can also be sorted according to the 
expression of a selected gene.

PCX2 supports many important extension to standard parallel coordinates such as use of transparent data lines, semi-transparent color bands to visualize cell selections, or dimensional zooming. Besides this also highlighting and animation of line traces is supported by PCX2. For more detailed information see $\left[\mathrm{RWK}^{+} 06\right]$.

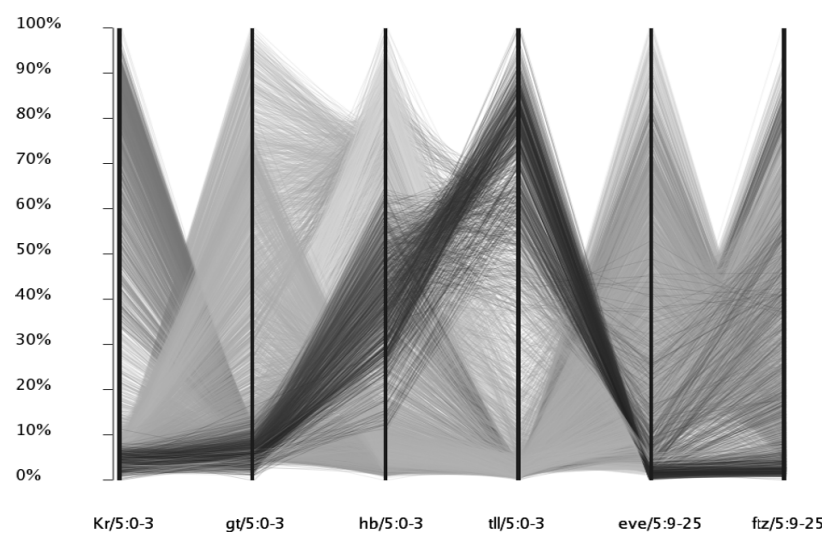

Figure 3: 2D Parallel coordinate view showing the expression of six selected genes in all blastoderm cells of the embryo (reproduced in color on p. 14).

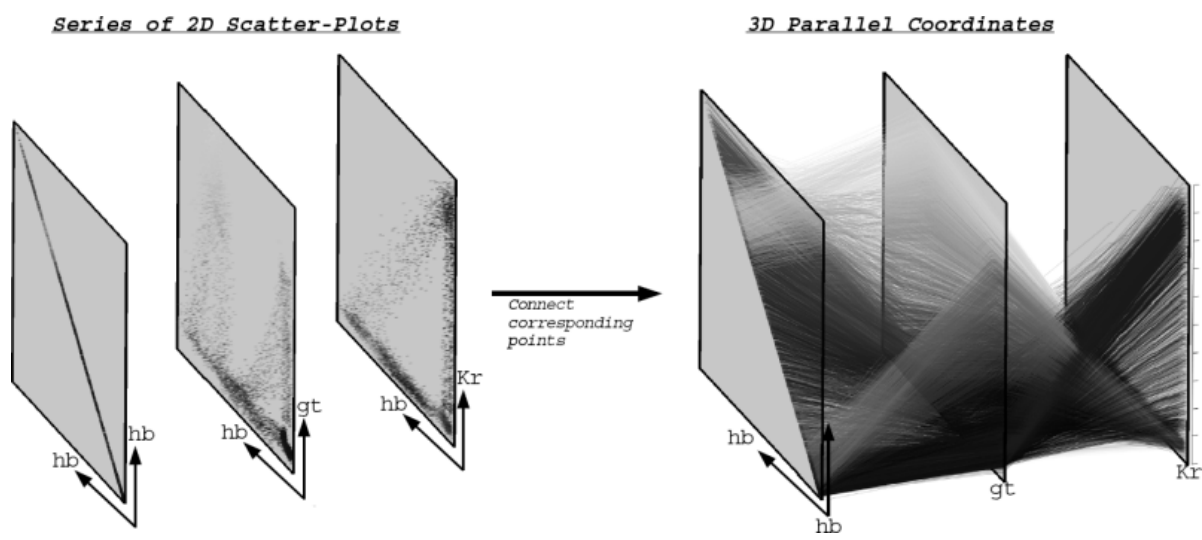

Figure 4: 3D Parallel coordinates are a direct extension to regular parallel coordinates and combine the principle of 2D-scatter-plots and 2D-parallel coordinates (reproduced in color on p. 14).

\subsection{Cell Magnifier}

Unlike all other views currently available in PCX2, the Cell Magnifier concentrates not on comparing gene expression values in different cells but on comparing expression values 


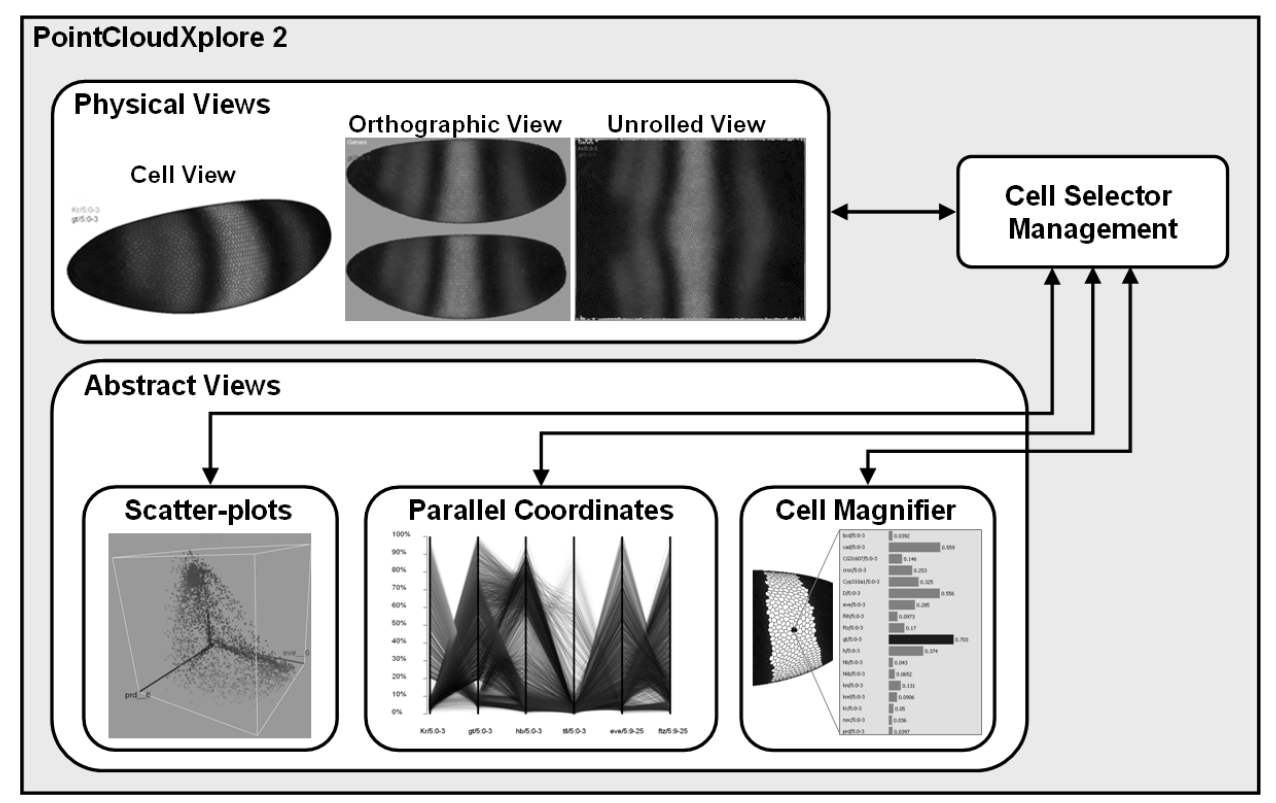

Figure 5: PointClouxXplore 2 system design. All views are linked via a central cell selector management system.

in just one cell. A bar graph is used to visualize gene expression values. Each gene is represented by one bar while the exact measured expression value is displayed in addition beside each bar. The cell to be displayed in the Cell Magnifier can be selected in any physical view and is highlighted by graying it out. An example illustrating use of the Cell Magnifier to analyze the expression in a cell located in the posterior giant expression domain will be provided later in Figure 8. For more detailed information about the Cell Magnifier see Weber et al. [WRH $\left.{ }^{+} 07\right]$.

\section{Linking the Views via Cell Selection}

The different views described in the previous section are useful in their own right and can be used individually to mine data sets for new information. It is, however, often useful to compare and correlate the information of different views. The principle of cell selection (also referred to as brushing) provides effective means for linking different views. By selecting cells of interest in one view and highlighting the same cell subset in all other views interactive exploration of the data becomes possible. Selection of cells can be performed in each view of PCX2 with respect to different data properties, e.g., cell position or gene expression (see Section 4.1). To allow for definition of more complex cell queries, cell selections can be combined using logical operations (see Section 4.2). 
In PCX2 all cell selections are stored in dedicated so called cell selector objects. All cell selectors are stored and managed in a central cell selector management system. All views have access to the same set of cell selectors via this central management system. This simple but effective system design allows one to easily integrate new views in PCX2 and link them to the already existing views. Figure 5 illustrates the basic system design of PCX2. The different views are each independent of one another and are interconnected only indirectly via the central cell selector management. Independent of the criteria employed to perform cell selection, each cell selector defines for each cell whether it is the selected or not. In addition, each cell selector is required to have a name, color, and a flag defining whether the cell selector should be displayed or not. Therefore, a common base class can be used to define properties common for all types of cell selectors. By using such a common interface all types of cell selectors can be efficiently displayed in all views. Which types of cell selectors can be edited and created in the different views then depends on according to which data properties cell selection can be performed in a view.

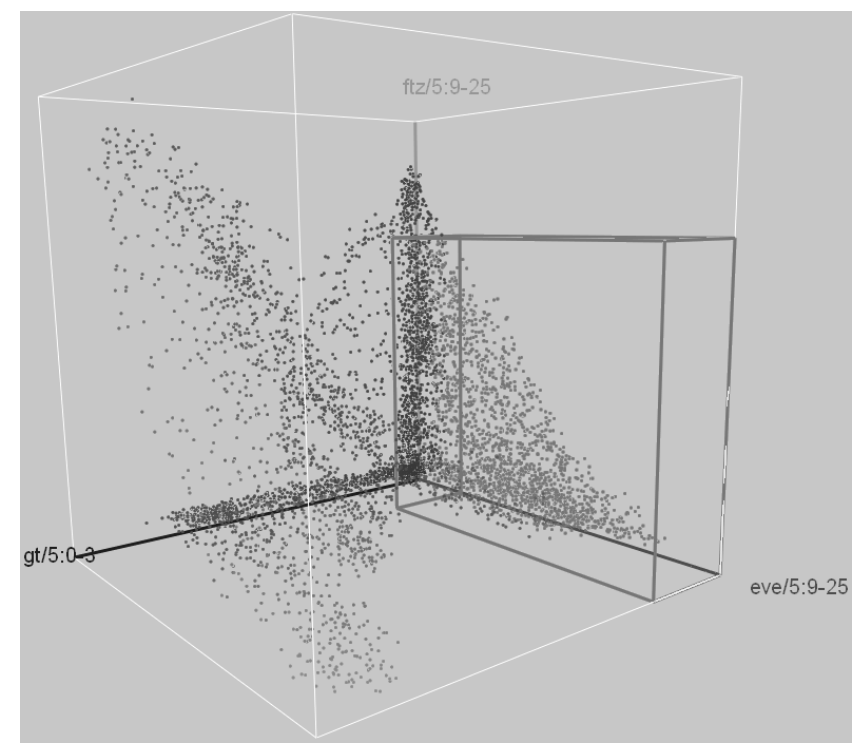

Figure 6: By drawing an axes aligned box in a scatter-plot one can select cells with respect to the expression of up to three genes (reproduced in color on p. 14).

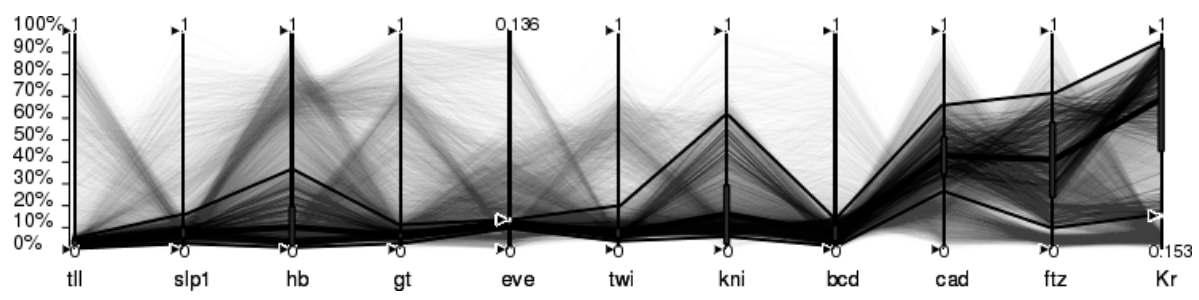

Figure 7: By defining ranges in expression using handles attached to each parallel axis it is possible to select cells with respect to the expression of all genes displayed in the parallel coordinate view. 


\subsection{Basic Cell Selection Operations}

Cell selection can be performed with respect to different data properties in different views. The basic cell selection operations performed directly by the user can be subdivided into three different categories:

- Cell selection based on cell positions can be performed in any physical view. By drawing on the surface of the embryo the researcher can define which cells in which region of the embryo are of interest.

- Cell selection based on gene expression can be performed in scatter-plots as well as parallel coordinates. Dedicated cell selectors allow the user to select cells with respect to ranges in gene expression. All cells contained in a so defined multidimensional box in gene expression space are marked as selected. In a scatter-plot cell selection can be performed with respect to the expression of up to three genes by defining an axis-aligned box in the plot (see Figure 6). In parallel coordinates cell selection can be performed with respect to all $n$ displayed data dimensions by defining ranges in gene expression with sliders attached to each parallel axis (see Figure 7).

- Cell selection based on spatial information and gene expression can be performed using seed cell selection and the Cell Magnifier. Seed cell selection requires the index of a cell as well a set of expression range(s) for selected genes as input. Using the cell currently displayed in the Cell Magnifier as seed point to initiate the selection process, a flood fill method is used to identify all cells in a contiguous region whose expression levels lie within the specified expression range(s). In the example shown in Figure 8, seed cell selection is used to select the posterior expression domain of the giant expression pattern. Besides seed cell selection, one can also use 3D parallel coordinates to select cells with respect to spatial information as well as gene expression. If data lines are sorted in 3D parallel coordinates with respect to AP- or DV-cell positions then ranges in AP/DV-space can be used in addition to range(s) in gene expression to perform cell selection.

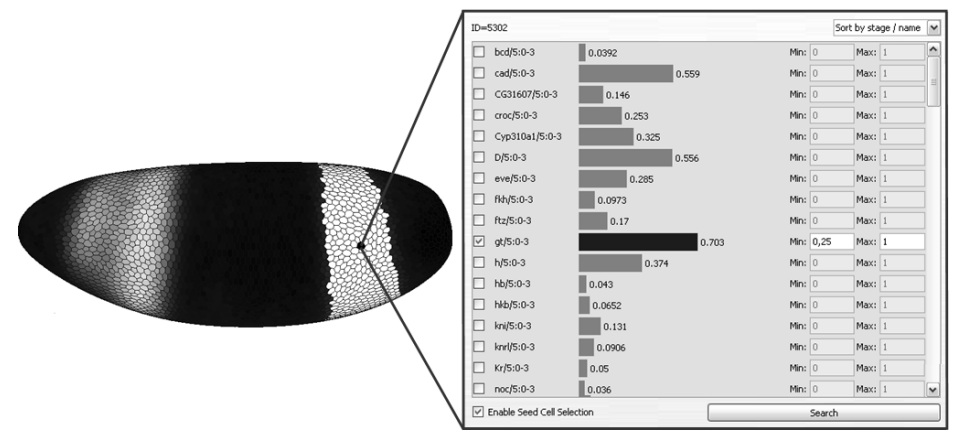

Figure 8: Using seed cell selection it is possible to easily and accurately select contiguous regions on the embryo that show specific expression properties. 


\subsection{Combining Cell Selections}

To make definition of more complex cell queries possible, basic cell selections can be combined in PCX2 using logical operations such as AND, OR, and NOT. The AND-operator defines the intersection of two cell selections and the OR-operator their union. The NOToperator then inverts a cell selection and selects all cells not selected by the input cell selection. Logical operators are implemented in PCX2 as special types of cell selectors that define a new selection of cells based on the cell selection defined by one or two other cell selectors. A logical operator can again function as input to another logical operator allowing complex cell queries to be defined. Such cell queries consisting of both basic and logical cell selectors can be represented as a tree structure in which the basic cell selectors are always leaves of the tree. In Figure 9, cell selectors are used to define the patterns of the genes giant ( $g t)$, hunchback $(h b)$, and Krüppel $(K r)$ via thresholding. The thresholded patterns are then combined using logical operators in order to validate a model for regulation of stripe two of the eve expression pattern. A comparison of the defined selection with the thresholded pattern of the gene eve shows a close match in the domain of eve stripe two indicating that $g t, h b$, and $K r$ may be able to regulate this part of the eve pattern.

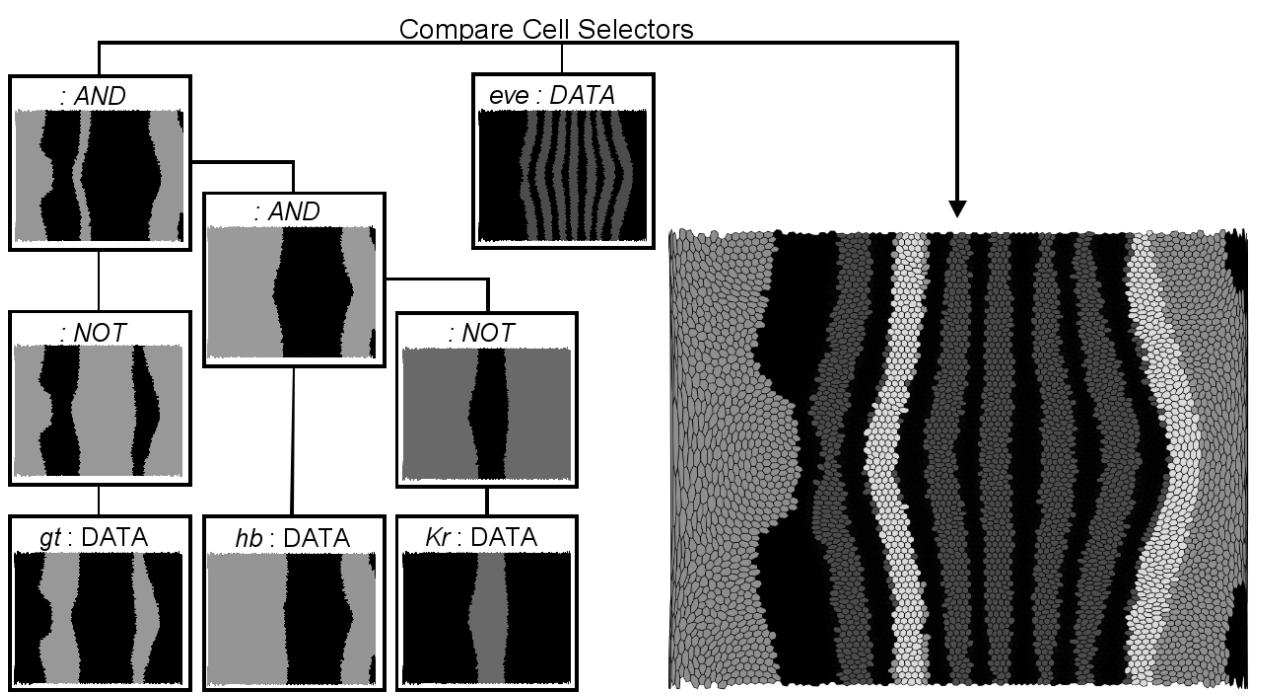

Figure 9: Combining cell selectors defining the patterns of $g t, h b$, and $K r$ using logical operators. The selection result is then compared with a cell selector that selects all cells expressing eve at medium to high levels by defining the overlay of both selections (reproduced in color on p. 14).

\section{Implementation}

PCX2 is an interactive exploration tool. All here described views are rendered interactively and all described interactions take, at most, a fraction of a second to complete. PCX2 
is implemented as $\mathrm{C}++$ stand-alone application using Trolltechs Qt 4.2 library (http: //www.trolltech.com/) and OpenGL (http://www.opengl.org/) as crossplatform widget and graphics libraries. PCX2 is available for Linux, MacOS and Windows. An earlier version of PCX has already been released to the public and can be downloaded free of charge from the BDTNPs web page http://bdtnp.lbl.gov/ $\mathrm{Fly}$-Net/bioimaging.jsp?w=pcx. The full version of PCX2 as described here will also be made available for free soon via the BDTNPs web page. PCX2 is used as a means to view the BDTNP's release data sets describing spatial gene expression in Drosophila blastoderms [BDT].

\section{Conclusions and Future Work}

PCX has already shown to be a valuable tool to members of the BDTNP as well as users of the publicly available 3D gene expression database of the BDTNP. Along with the next release of the database we will also make PCX2 available for free public download. PCX2 provides all features of PCX and includes additional extension such as 3D parallel coordinates and supports visualization of the latest 3D gene expression data created by the BDTNP. Scatter-plots and parallel coordinates should allow researchers to examine relationships between 20-30 genes.

Here we have provided an overview of all views available in PCX2 and shown how linking of the views via cell selection can be used for effective data exploration. Using selected examples, we have shown how one can analyze the spatial expression pattern of genes using physical views and expression surfaces. Information visualization views, such as scatterplots and parallel coordinates, then enable analysis of gene inter-relationships directly in gene expression space. We have illustrated how dedicated cell selection operations such as seed cell selection can be used to effectively define cells of interest, such as an expression domain of a selected gene. We have also shown how binary logical models of genetic interactions can be analyzed by combining thresholded patterns of selected genes using logical operations.

As the BDTNP continues to collect data for many more genes, this imposes several new challenges for future work. For example, with our current approach it is possible to effectively visualize expression levels of five to six genes simultaneously in a physical view. (The exact number depends on the spatial distribution of the patterns.) Mapping an even larger number of genes to color will be one of the challenges arising from the increasing number of genes. By integrating automated data analysis methods, such as clustering and dedicated dimension reduction techniques into PCX, we hope to address the arising need to examine the expression of several hundred genes.

\section{Acknowledgment}

This work was supported by the National Institutes of Health through grant GM70444, by the National Science 
Foundation through award ACI 9624034 (CAREER Award) as part of the Large Scientific and Software Data Set Visualization (LSSDSV) program under contract ACI 9982251, by the LBNL Laboratory Directed Research Development (LDRD) program; and by a large Information Technology Research (ITR) grant. Work at Lawrence Berkeley National Laboratory is conducted under Department of Energy contract DE-AC02-05CH11231 We thank the members of the Visualization and Computer Graphics Research Group at the Institute for Data Analysis and Visualization (IDAV) at the University of California, Davis; the members of the BDTNP at the Lawrence Berkeley National Laboratory (LBNL), the members of the Visualization Group at LBNL, and the International Research Training Group 1131 at the University of Kaiserslautern.

\section{References}

[BDT] BDTNP. http://bdtnp.lbl.gov/Fly-Net/bioimaging.jsp.

[BWK00] M. Q. Wang Baldonado, A. Woodruff, and A. Kuchinsky. Guidelines for using multiple views in information visualization. In AVI '00: Proceedings of the working conference on Advanced visual interfaces, pages 110-119, New York, NY, USA, 2000. ACM Press.

[DGH03] H. Doleisch, M. Gasser, and H. Hauser. Interactive Feature Specification for Focus+Context Visualization of ComplexSimulation Data. In G.-P. Bonneau, S. Hahmann, and C. D. Hansen, editors, Data Visualization 2003 (Proceedings of the Eurographics/IEEE TCVG Symposimon Visualization), 2003.

$\left[\mathrm{FLK}^{+}\right.$05] C. C. Fowlkes, C. L. Luengo Hendriks, S. V. E. Keränen, M. D. Biggin, D. W. Knowles, D. Sudar, and J. Malik. Registering Drosophila embryos at cellular resolution to build a quantitative3d map of gene expression patterns and morphology. In CSB 2005 Workshop on BioImage Data Minning and Informatics, August 2005.

$\left[\mathrm{FLK}^{+}\right.$07] C. C. Fowlkes, C. L. Luengo Hendriks, S. V. E. Keränen, G. H. Weber, O. Rübel, M.-Y. Huang, S. Chatoor, L. Simirenko, M. B. Eisen, B. Hamann, D. W. Knowles, M. D. Biggin, and J.Malik. Constructing a quantitative spatio-temporal atlas of gene expression in Drosophila blastoderm. to appear in: Cell (Accepted January 2008).

[FWR99] Y.-H. Fua, M. O. Ward, and E. A. Rundensteiner. Hierarchical parallel coordinates for exploration of large datasets. In IEEE Visualization 1999, pages 43-50. IEEE Computer Society Press, 1999.

$\left[\mathrm{GRW}^{+} 00\right]$ D. L. Gresh, B. E. Rogowitz, R. L. Winslow, D. F. Scollan, and C. K. Yung. WEAVE: A system for visually linking 3-D and statistical visualizations, applied to cardiac simulation and measurement data. In Thomas Ertl, Bernd Hamann, and Amitabh Varshney, editors, Proceedings IEEE Visualization 2000, pages 489-492, Los Alamitos, CA, USA, 2000. IEEE Computer Society Press.

[Hen98] Chris Henze. Feature detection in linked derived spaces. In David Ebert, Holly Rushmeier, and Hans Hagen, editors, Proceedings IEEE Visualization '98, pages 87-94, Los Alamitos, CA, USA, 1998. IEEE Computer Society Press.

[HLD02] Helwig Hauser, Florian Ledermann, and Helmut Doleisch. Angular Brushing of Extended Parallel Coordinates. In IEEE Symposium on Information Visualization (InfoVis'02), pages 127-130. IEEE Computer Society Press, 2002.

[Ins84] Alfred Inselberg. Parallel Coordinates for Multidimensional Displays. In Spatial Information Technologies for Remote Sensing Today and Tomorrow, The Ninth William T. Pecora Memorial Remote Sensing Symposium, pages 312-324. IEEE Computer Society Press, 1984.

$\left[\mathrm{KFL}^{+}\right.$06] S. V. E. Keränen, C. C. Fowlkes, C. L. Luengo Hendriks, D. Sudar, D. W. Knowles, J. Malik, and M. D. Biggin. Three-Dimensional Morphology and Gene Expression in the Drosophila blastoderm at Cellular Resolution I: Dynamics. Genome Biology, 7(12):R124, 2006. 
[KSH04] R. Kosara, G. N. Sahling, and H. Hauser. Linking Scientific and Information Visualization with Interactive 3D Scatterplots. In Short Communication Papers Proceedings of the 12th International Conferencein Central Europe on Computer Graphics, Visualization, and Computer Vision (WSCG), pages 133-140, 2004.

[Law92] P. A. Lawrence. The Making of a Fly: The Genetics of Animal Design. Blackwell Publishing, Inc., 1992.

$\left[\mathrm{LKF}^{+}\right.$06] C. L. Luengo Hendriks, S. V. E. Keränen, C. C. Fowlkes, L. Simirenko, G. H. Weber, A. H. DePace, C. Henriquez, D. W. Kaszuba, B. Hamann, M.l B. Eisen, J. Malik, D. Sudar, M. D. Biggin, and D. W. Knowles. Three-Dimensional Morphology and Gene Expression in the Drosophila blastoderm at Cellular Resolution I: Data Acquisition Pipeline. Genome Biology, 7(12):R123, 2006.

[PKH04] H. Piringer, R. Kosara, and H. Hauser. Interactive Focus+Context Visualization with Linked 2D/3D Scatterplots. In Proceedings of the Second International Conference on Coordinated \& Multiple Views in Exploratory Visualization (CMV'04), pages 49-60, Washington, DC, USA, 2004. IEEE Computer Society.

$\left[\right.$ RWK $^{+}$06] O. Rübel, G. H. Weber, S. V. E. Keränen, C. C. Fowlkes, C. L. Luengo Hendriks, L. Simirenko, N. Y. Shah, M. B. Eisen, M. D. Biggin, H. Hagen, J. D. Sudar, J. Malik, D. W. Knowles, and B. Hamann. PointCloudXplore: Visual analysis of 3D gene expression data using physical views and parallel coordinates. In B. Sousa Santos, T. Ertl, and K.I Joy, editors, Data Visualization 2006 (Proceedings of EuroVis 2006), pages 203-210, Aire-la-Ville, Switzerland, 2006.

[Weg90] Edward J. Wegman. Hyperdimensional Data Analysis Using Parallel Coordinates. Journal of the American Statistical Association, 85(411):664-675, September 1990.

[WL97] Edward J. Wegman and Qiang Luo. High dimensional clustering using parallel coordinates and the grand tour. Computing Science and Statistics, 28:361-368, 1997.

[WRH ${ }^{+}$07] G. H. Weber, O. Rübel, M.-Y. Huang, A. H. DePace, C. C. Fowlkes, S. V. E. Keränen, C. L. Luengo Hendriks, H. Hagen, D. W. Knowles, J. Malik, M. D. Biggin, and B. Hamann. Visual Exploration of Three-dimensional Gene Expression Using Physical Views and Linked Abstract Views. Accepted for Publication in IEEE Transactions on Computational Biology and Bioinformatics, 2007. 


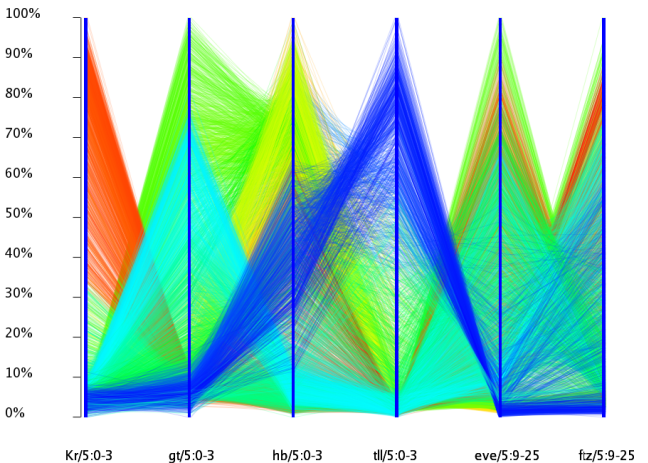

Figure 3: 2D Parallel coordinate view showing the expression of six selected genes in all blastoderm cells of the embryo.

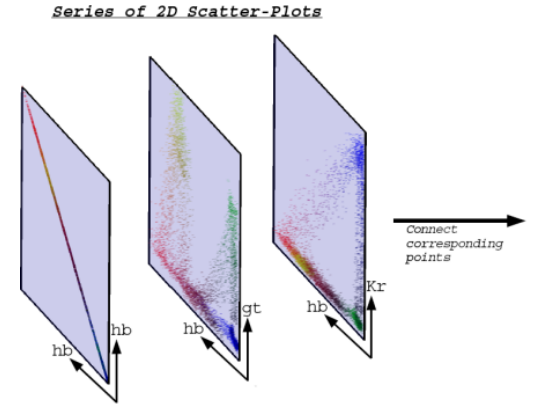

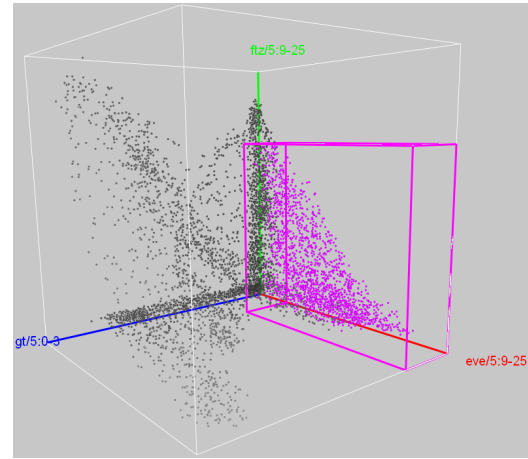

Figure 6: By drawing an axes aligned box in a scatter-plot one can select cells with respect to the expression of up to three genes.

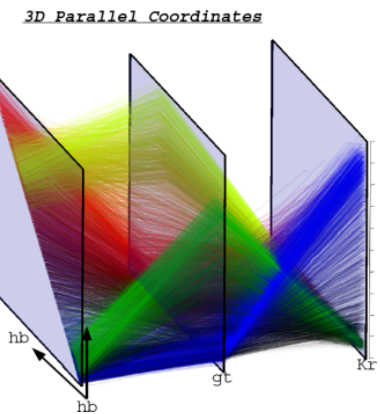

Figure 4: 3D Parallel coordinates are a direct extension to regular parallel coordinates and combine the principle of 2D-scatter-plots and 2D-parallel coordinates.

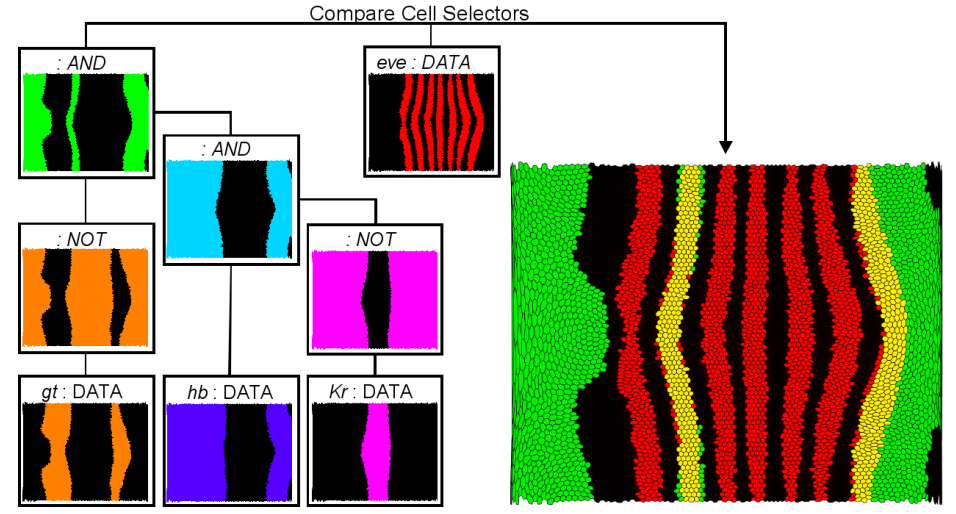

Figure 9: Combining cell selectors defining the patterns of $g t, h b$, and $K r$ using logical operators. The selection result is then compared with a cell selector that selects all cells expressing eve at medium to high levels by defining the overlay of both selections. 